\title{
Fréquence, types et déterminants des complications dégénératives du diabète de type 2 au Maroc : données d'inclusion de la cohorte « EpiDiaM»
}

\author{
Z. Selihi, ${ }^{1,2}$ M. Berraho, ${ }^{1}$ K. El Rhazi, ${ }^{7}$ Y. El Achhab, ${ }^{7}$ B. Lyoussi ${ }^{2}$ et C. Nejjari ${ }^{1}$
}

$$
\begin{aligned}
& \text { معدل انتشار وأنماط ومحدِّدات مضاعفات التشيخ للسكري من النمط } 2 \text { في المغرب: بيانات "EpiDiaM" المشتملة على الأتراب }
\end{aligned}
$$

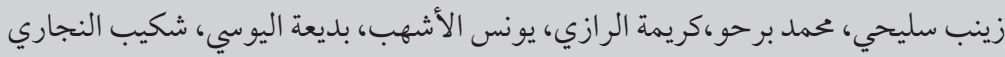

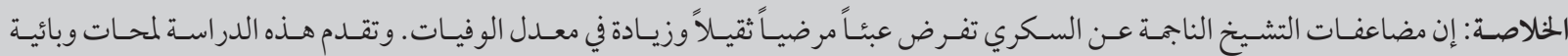

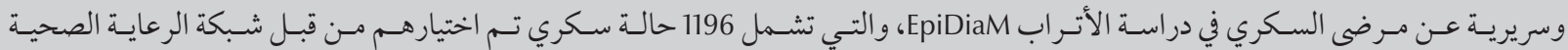

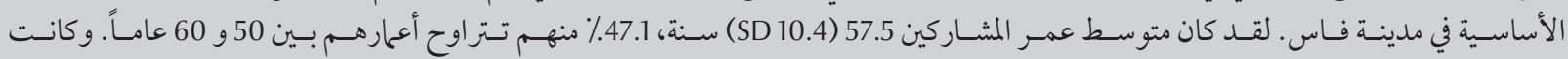

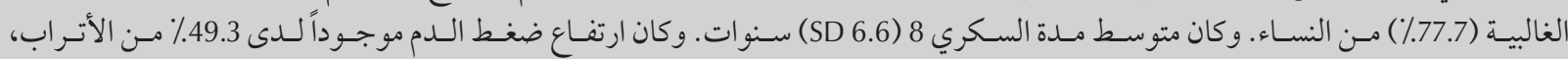

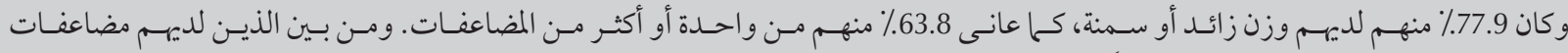

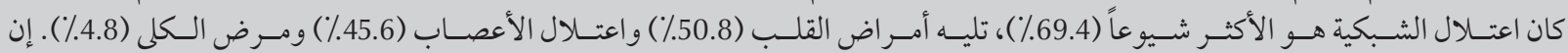

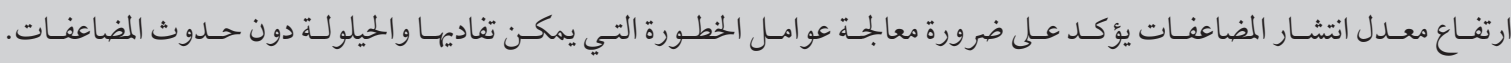

RÉSUMÉ Le diabète est une maladie à lourde charge de morbidité et de mortalité liées aux complications dégénératives. La présente étude présente le profil épidémiologique et clinique des patients diabétiques inclus dans l'étude de cohorte «EpiDiaM», qui portait sur 1196 cas recrutés au niveau du réseau de soins de santé de base de la ville de Fès. L'âge moyen des participants était de 57,5 (ET 10,4) ans. Dans 47,1 \% des cas, les patients étaient âgés entre 50 et 60 ans. La majorité $(77,7 \%)$ était des femmes. L'ancienneté moyenne du diabète était de 8 (ET 6,6) ans. L'hypertension était présente chez 49,3\% des patients alors que 77,9\% étaient en surpoids ou obèses ; $63,8 \%$ des patients souffraient de complications. Chez ces derniers, la rétinopathie était la complication la plus fréquente $(69,4 \%)$, suivie par la cardiopathie (50,8\%), la neuropathie (45,6 \%) et la néphropathie (4,8\%). La forte prévalence des complications souligne la nécessité d'agir sur les facteurs de risque évitables et de prévenir les complications.

Frequency, types and determinants of degenerative complications of type 2 diabetes in Morocco: "EpiDiaM" cohort inclusion data

ABSTRACT Degenerative complications as a result of diabetes impose a heavy disease burden and increase mortality. This study presents epidemiological and clinical profiles of diabetic patients in the EpiDiaM cohort study, which include 1196 diabetic cases recruited from the basic health care network in the city of Fez. The mean age of the participants was 57.5 (SD 10.4) years with $47.1 \%$ aged between 50 and 60 years. The majority (77.7\%) were women. The mean duration of diabetes was 8 (SD 6.6) years. Hypertension was found in $49.3 \%$ of the cohort, $77.9 \%$ were overweight or obese and $63.8 \%$ had one or more complications. Among those with complications, retinopathy was the most common (69.4\%), followed by heart conditions (50.8\%), neuropathy $(45.6 \%)$ and renal disease $(4.8 \%)$. The high prevalence of complications emphasizes the need to address the avoidable risk factors and prevent complications.

'Laboratoire d'Épidémiologie, Recherche Clinique et Santé Communautaire, Faculté de Médecine et de Pharmacie, Fès (Maroc) (Correspondance à adresser à Z. Selihi : selihi.7zineb@gmail.com). ${ }^{2}$ Laboratoire de Physiologie, Pharmacologie et Santé Environnementale, Faculté des Sciences Dhar ElMehraz, Fès (Maroc).

Reçu : 02/10/14 ; accepté : 12/03/15 


\section{Introduction}

Le diabète est une maladie à lourde charge de morbidité et de mortalité liées aux complications dégénératives (1). Nombre de complications du diabète peuvent être évitées ou retardées par des mesures de prévention et des programmes de prise en charge de cette maladie, notamment en diagnostiquant rapidement le diabète, en informant efficacement les patients et les professionnels et en fournissant un ensemble de soins sur le long terme. En effet, les actions et les mesures de prévention ciblant les facteurs de risque responsables de la survenue des complications du diabète imposent leurconnaissance et leuridentification.

Au Maroc, pays en pleine phase de transition démographique, nutritionnelle et épidémiologique $(2,3)$, la fréquence $\mathrm{du}$ diabète est en augmentation, avec plus d'un million et demi de diabétiques de type 2 en 2010 (4). Ce chiffre atteindrait 2,5 millions à l'horizon 2030 (4).

Pour déterminer les facteurs de risque de survenue de complications du diabète chez le diabétique marocain, nous avons mis en place, en 2012, une étude de type cohorte «EpiDiaM $»$. Dans la présente étude, nous présentons les données de l'inclusion des patients diabétiques sur le profil épidémiologique et clinique.

\section{Méthodes}

\section{Population et type d'étude}

L'étude a porté sur une série de 1196 cas inclus dans la cohorte «EpiDiaM ». L'inclusion des patients diabétiques de type 2 s'est déroulée du mois d'avril 2012 au mois de janvier 2013 au niveau de cinq centres de santé du réseau de soins de santé de base et d'un centre de référence de prise en charge du diabète dans la ville de Fès.

\section{Recueil de l'information}

Le recueil de l'information s'est fondé sur un questionnaire administré en face à face par des enquêteurs formés et complété à partir du dossier médical de chaque patient. Six axes ont été explorés : connaissances générales sur le diabète, alimentation, complications, traitements, auto-surveillance et suivi. En plus du questionnaire, nous avons mesuré pour chaque patient le poids, la taille et la tension artérielle. Les femmes enceintes et les diabétiques de type 1 ont été exclus de l'étude.

\section{Résultats}

L'âge moyenétait de 57,5 ans (écart-type [ET] : 10,4). Dans 47,1\% des cas, les patients étaient âgés entre 50 et 60 ans. La majorité de notre population était de sexe féminin $(77,7 \%)$. L'ancienneté moyenne du diabète était de 8 ans (ET: 6,6). L'hypertension était présente chez $49,3 \%$ des patients tandis que $77,9 \%$ des patients étaient en surpoids ou obèses. Parmi les 1196 cas diabétiques observés, 1017 patients ont réalisé un test d'HbAlc dans les trois derniers mois et 79,8\% d'entre eux étaient en déséquilibre glycémique.

Parmi les diabétiques sélectionnés, $36,2 \%$ ne souffraient d'aucune complication et $63,8 \%$ avaient une ou plusieurs complications. Chez $33,4 \%$ de nos patients diabétiques, le diagnostic du diabète était concomitant avec le diagnostic de la complication, alors que $47,7 \%$ des cas ont développé des complications après le diagnostic du diabète.

Sur l'ensemble de nos patients diabétiques présentant des complications, $69,4 \%$ présentaient une complication ophtalmologique, diagnostiquée pour $65,0 \%$ des cas après le diagnostic du diabète et pour 13,7 \% concomitante au diagnostic du diabète. Parmi les mêmes patients, la cardiopathie était présente chez $50,8 \%$; elle s'est manifestée après le diagnostic du diabète pour $43,4 \%$ et pour $32,0 \%$ elle était concomitante au diagnostic du diabète. La neuropathie était présente chez $45,6 \%$ des patients : dans $71,3 \%$ des cas, elle est survenue après le diagnostic du diabète et dans 16,1\% elle était concomitante au diagnostic du diabète. La néphropathie était présente chez $4,8 \%$ des patients : dans $59,5 \%$ des cas, elle est survenue après le diagnostic du diabète et dans $21,6 \%$ avant même le diagnostic du diabète.

Dans le tableau 1, nous présentons la répartition des diabétiques selon la fréquence des complications et selon le sexe, l'âge et la durée de la maladie. Ainsi, $66,7 \%$ des complications sontsurvenues dans les cinq premières années suivant le diagnostic du diabète. La fréquence était de $69,9 \%$ pour les rétinopathies, $46,0 \%$ pour les neuropathies, 5,6\% pour les néphropathies et $45,7 \%$ pour les complications cardiaques.

\section{Discussion}

Nous avons mis en place une étude de type cohorte «EpiDiaM » pour déterminer les facteurs de risque de la survenue des complications du diabète chez les patients diabétiques de type 2 au Maroc. La présente étude concerne les données de linclusion de la cohorte «piDiaM».

Dans notre étude, $63,8 \%$ des diabétiques présentaient une ou plusieurs complications. Cette prévalence est considérée comme comparable aux taux de prévalence obtenus dans d'autres études similaires $[68,7 \%$ en Libye (5) et $60 \%$ en Algérie (6)].

Les données de l'inclusion ont permis de décrire les complications dégénératives chez les diabétiques de type 2 marocains en termes de fréquence, de localisation et d'association. De plus, cette étude a également montré que chez $33,4 \%$ de nos patients diabétiques, le diagnostic du diabète était concomitant avec le 


\begin{tabular}{|c|c|c|c|c|c|c|}
\hline \multirow[t]{2}{*}{ Variable } & \multicolumn{2}{|c|}{ Complications $^{\mathrm{a}}$} & \multicolumn{4}{|c|}{ Complications par localisation (\%) } \\
\hline & Nbre $^{a}$ & $\%$ & Néphropathie $^{b}$ & Rétinopathie $^{b}$ & Cardiopathie $^{\mathrm{b}}$ & Neuropathie \\
\hline Population totale & 763 & 63,8 & 4,8 & 69,4 & 50,8 & 45,6 \\
\hline \multicolumn{7}{|l|}{ Sexe } \\
\hline Femme $(n=930)$ & 618 & 66,5 & 6,3 & 69,6 & 53,9 & 47,7 \\
\hline Homme $(n=266)$ & 145 & 54,5 & 8,9 & 74,5 & 37,2 & 46,2 \\
\hline \multicolumn{7}{|l|}{ Tranche d'âge (ans) } \\
\hline$\leq 49(n=132)$ & 65 & 49,2 & 12,3 & 66,2 & 27,7 & 46,2 \\
\hline $50-59(n=564)$ & 363 & 47,5 & 6,1 & 71,6 & 48,7 & 48,5 \\
\hline $60 \leq(n=500)$ & 335 & 67,0 & 6,6 & 70,1 & 57,3 & 46,6 \\
\hline \multicolumn{7}{|c|}{ Durée de la maladie (ans) } \\
\hline$<5(n=453)$ & 302 & 66,7 & 5,6 & 69,9 & 45,7 & 46,0 \\
\hline $5-10(n=327)$ & 204 & 62,4 & 5,9 & 69,1 & 53,9 & 51,0 \\
\hline$\geq 10(n=406)$ & 256 & 63,0 & 8,2 & 72,3 & 54,3 & 46,5 \\
\hline
\end{tabular}

${ }^{a}$ Effectif, toutes complications confondues.

${ }^{b}$ La présence d'une complication n'est pas exclusive de la présence d'une autre.

diagnostic de la complication. Cela dénote le retard du diagnostic et le danger de cette maladie silencieuse, qui peut passer inaperçue pendant de longues années. Les données de l'étude UKPDS (United Kingdom Prospective Diabetes Study) ont montré que le début réel du diabète de type 2 précédait de 8 à 10 ans le diagnostic de la maladie (7). Parmi nos patients, 47,7\% ont développé des complications après le diagnostic du diabète ; $66,7 \%$ de ces complications sont apparues dans les cinq ans suivant le diagnostic du diabète.

La population de diabétiques souffrant déjà des complications nous permettra de monter une étude de cohorte rétrospective à la recherche des déterminants de la survenue des complications, en utilisantles différentes dates disponibles (date du diagnostic et dates de survenue des complications). La population des sujets ne présentant pas de complications représentera notre population de la cohorte prospective avec un suivi régulier tous les trois ans à la recherche des complications du diabète de type 2 .

\section{Conclusion}

Les résultats de l'inclusion de l'étude EpiDiaM ont révélé une très forte prévalence des complications dégénératives du diabète de type 2 dans la ville de Fès. À défaut de prévenir le diabète, il importe d'en prévenir les complications, dont les facteurs de risque sont évitables. La prévention reste un volet essentiel dans la prise en charge du diabète de type 2 . De nombreuses complications chez ces patients peuvent être évitées ou retardées grâce à une prise en charge multidisciplinaire préventive, qui s'appuie sur la lutte contre les facteurs de risque et l'amélioration du contrôle de la maladie.

\section{Remerciements}

Nous remercions la Faculté de Médecine et de Pharmacie de Fès ainsi que l'Association des Diabétiques de la Wilaya de Fès pour leur soutien financier à cette étude.

Conflits d'intérêts : Les auteurs ne déclarent aucun conflit d'intérêt.

\section{Références}

1. Journée mondiale du DIABÈTE2012: 80 \% du fardeau liésà ses complications - WDD-NHS (http://blog.santelog.com/2012/11/14/ journee-mondiale-du-diabete-2012-80-du-fardeau-lies-a-sescomplications-wdd-nhs/, consulté le 24 avril 2105).

2. Amuna P, Zotor FB. Epidemiological and nutrition transition in developing countries: impact on human health and development. Proc Nutr Soc. 2008 Feb;67(1):82-90.

3. Benjelloun S. Nutritional transition in Morocco. Public Health Nutr. 2002;5:135-40

4. Shaw JE, Sicree RA, Zimmet PZ. Global estimates of the prevalence of diabetes for 2010 and 2030. Diabetes Res Clin Pract. 2010 Jan;87(1):4-14.
5. Roaeid RB, Kadiki OA. Prevalence of long-term complications among type 2 diabetic patients in Benghazi, Libya. J Diabetol. 2001 Oct;3:5.

6. Zaoui S, Biémont C, Meguenni K. Approche épidémiologique du diabète en milieux urbain et rural dans la région de Tlemcen (Ouest algérien). Cahiers d'études et de recherches francophones / Santé. 2007, 17(1):15-21. DOI : 10.1684/ san.2007.0060.

7. UK Prospective Diabetes Study 6. Complications in newly diagnosed type 2 diabetic patients and their association with different clinical and biochemical risk factors. Diabetes Res. 1990 Jan;13(1):1-11. 